\title{
New model of conjunctival scarring in the mouse eye
}

Martin B Reichel, M Francesca Cordeiro, Robert A Alexander, Ian A Cree, Shomi S Bhattacharya, Peng T Khaw

\begin{abstract}
Aims-To establish a simple model of conjunctival wound healing in the mouse eye.

Methods-4 week old BALB/c mouse eyes were studied over a 14 day period. Surgical procedure under general anaesthesia involved a blunt dissection of the conjunctiva performed by injection of $25 \mu$ of PBS via a 27 gauge needle into one eye, while the contralateral eye was used as control. Mice were assessed clinically and sacrificed at $1,2,3,7$, and 14 days after surgery. Enucleated eyes were prepared for histological analysis. Development of scar tissue was studied with haematoxylin and eosin, oxidation aldehyde fuchsin, and van Gieson stains, with assessment of cellularity, extracellular matrix formation, and wound characterisation.
\end{abstract}

Results-Histological analysis revealed a marked and characteristic healing response initiated by a predominantly granulocytic inflammatory reaction at day 1 with peak fibroblast activity 3 days after surgery. Oxytalan fibres and newly laid collagen fibres were detected early in the subconjunctival wound area and up to 7 days after surgery. Remodelling and complete organisation of scar tissue was evident by day 14 .

Conclusion-A single subconjunctival injection in the mouse eye results in a marked and consistent healing response. This represents a simple, inexpensive, and reliable animal model of conjunctival scarring. The mouse is a biologically well characterised animal model and allows the use of a wide variety of molecular tools. There are potentially significant clinical applications, in particular in investigating the effects of modulating agents such as antimetabolites, growth factors, and their antagonists on conjunctival scarring.

(Br F Ophthalmol 1998;82:1072-1077)

Pathology, Institute of Ophthalmology, Bath

Street, London

I A Cree

Correspondence to: Mr P T Khaw, Wound Healing Research Unit, Department of Pathology and Glaucoma, Institute of Ophthalmology and Moorfields Eye Hospital, Bath Street, London EC1V9EL

Accepted for publication 24 March 1998 surgery, such as glaucoma, pterygia, and strabismus. In glaucoma filtration surgery, fail- ure is most often due to scarring at the subconjunctival level at the bleb and sclerostomy sites. $^{1-3}$

Various antimetabolites have been investigated as modulators of the conjunctival scarring response in an attempt to improve the results of filtration surgery. The potent antiscarring effects of mitomycin $\mathrm{C}$ and 5-fluorouracil have been studied in vitro ${ }^{4}$ and in vivo using primarily rabbit and monkey models of glaucoma filtration surgery. ${ }^{5-7}$ Other less common animal models of filtration surgery, developed to investigate the processes of wound healing in the sclera and conjunctiva, include dogs, cats, and rats. ${ }^{8-11}$

Fistulising surgery, however, is a complex process of wound healing because of the presence of aqueous humour causing dynamic changes in the local environment and the breakdown of the blood-aqueous barrier. In addition, aqueous humour contains various cytokines and chemical mediators that greatly influence the local cellular response. ${ }^{12}$ These factors make it difficult to assess the conjunctival wound healing response on its own. It was the aim of this study to develop a simple conjunctival scarring model to investigate specifically the conjunctival component of the wound healing process.

We chose the mouse as opposed to other species, such as the rabbit, because the mouse is a well characterised model on which a wide variety of readily available molecular tools and reagents may be used. Using histological techniques that identify collagen, oxytalan, elaunin, and mature elastic fibres, we have demonstrated a reproducible and characteristic conjunctival healing response in the mouse eye.

\section{Materials and methods}

Twenty 4 week old BALB/c mice underwent general anaesthesia with intraperitoneal injections of $0.2 \mathrm{ml}$ Hypnorm (Janssen Pharmaceutical Ltd, Oxford), and Hypnovel (Roche, Welwyn Garden City) mixed 1:1:6 with distilled water. A blunt dissection of the subconjunctival space was performed by injection of $25 \mu 1$ of sterile phosphate buffered saline (PBS, pH 7.34 and osmolality $0.15 \mathrm{~mol}$ ) as measured in a $1 \mathrm{ml}$ syringe (Terumo, Myjector $100 \mathrm{u}$ ) before delivery, for each injection. This syringe has a 27 gauge needle, and the measured volume of PBS when injected into one eye produced a visible subconjunctival bleb (Fig 1A). The contralateral, untreated eye was used as the control. Visualisation was with an OPMI MDU Zeiss microscope. The subconjunctival bleb was formed at the same position in each 
animal for ease of identification- $0.5 \mathrm{~mm}$ behind the limbus and as close as possible to the posterior canthal angle. This position was marked with a corneal $10-0$ nylon suture at post mortem but before enucleation. Mice were assessed clinically and four each per time point were sacrificed by cervical dislocation at $1,2,3,7$, and 14 days after surgery. All experiments were performed in adherence to the Declaration of Helsinki and The Guiding Principles in the Care and Use of Animals (DHEW Publication, NHI 80-23).

Enucleated eyes were prepared for histological analysis after fixation for 24 hours with $10 \%$ buffered formaldehyde and embedded whole in paraffin wax. Development of scar tissue was studied in sequential $5 \mu \mathrm{m}$ thick sections using the following special stains: haematoxylin and eosin to assess the inflammatory reaction, van Gieson to demonstrate collagen deposition, and aldehyde fuchsin for elastic and elaunin fibres together with oxidationaldehyde fuchsin for additionally demonstrating oxytalan fibres. ${ }^{13}$ Oxytalan fibres are a member of the elastic fibre family of connective tissue. Their presence is characteristic of the earliest identifiable stage of elastogenesis indicating local extracellular matrix production and deposition. Oxytalan fibres are differentiated from mature elastic fibres by their characteristic staining following the oxidation stage of aldehyde fuchsin. Elaunin fibres represent an intermediary stage of development of between oxytalan and mature elastic fibres.

Grading of the inflammatory response was performed by a masked observer (IAC), using a semiquantitative five point system of analysis. Assessment of extracellular matrix components was also assessed using a semiquantitative five point system of analysis, and performed by a masked observer (RAA). Total cell number within the wound site was assessed quantitatively using Kalcium Analyse (Kinetic Image Limited, Liverpool) and Image-Pro® Plus (Media Cybernetics, Silver Spring) image analysis software. Statistical analysis was performed using the Student's paired $t$ test comparing operated and control eyes, and using ANOVA (SPSs) to compare treatment groups with respect to cellularity and the presence of extracellular matrix components within the subconjunctival bleb area.

\section{Results}

A subconjunctival bleb was visible in all eyes following the subconjunctival injection and was macroscopically apparent until 24 hours (Fig 1B). Thereafter thickening of the conjunctiva at the surgical site decreased with time but was clearly evident histologically until day 7 . At 14 days, the macroscopic appearance of the eye that had received a subconjunctival injection was similar to that of the control eye (Fig 1C). There was no clinical evidence of periocular inflammation at any time after surgery, nor was there any macroscopic or histological evidence of intraocular inflammation in injected eyes throughout the follow up period.

Histological evaluation of the effect of the subconjunctival injection showed a typical re-
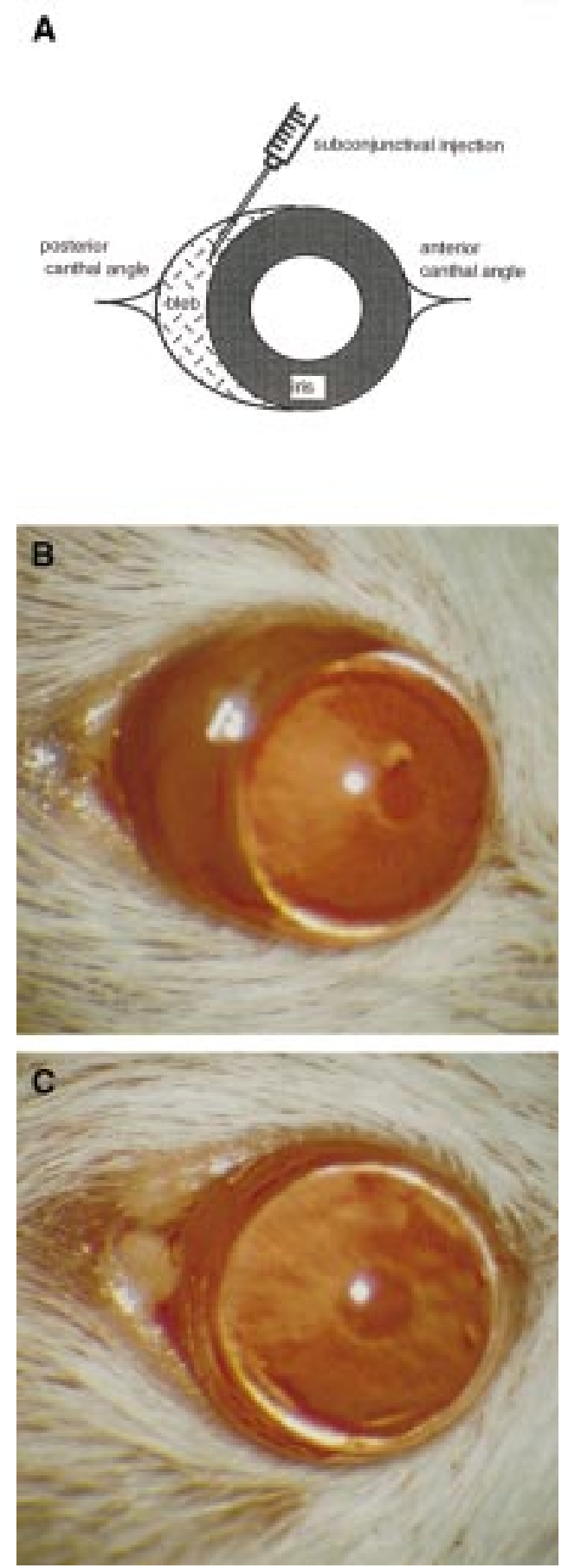

Figure 1 Subconjunctival injections of $25 \mu$ of PBS were given $0.5 \mathrm{~mm}$ behind the limbus and in close proximity to the posterior canthal angle $(A)$ of each mouse eye. $A$ subconjunctival bleb was visible in all eyes following the subconjunctival injection and was macroscopically apparent until 24 hours (B). At 14 days, the macroscopic appearance of the eye that had received a subconjunctival injection was similar to that of the control eye $(C)$.

sponse to injury. Figures $2 \mathrm{~A}-\mathrm{C}$ show haematoxylin and eosin stained sections at different time points after surgery and demonstrate the cellular changes associated with development of the scarring response. Compared with the control eye (Fig 2A), there was histological evidence of a marked healing response lasting up to day 14. An initial influx of granulocytes (neutrophil polymorphonuclear leucocytes), seen in association with considerable oedema of the 
A
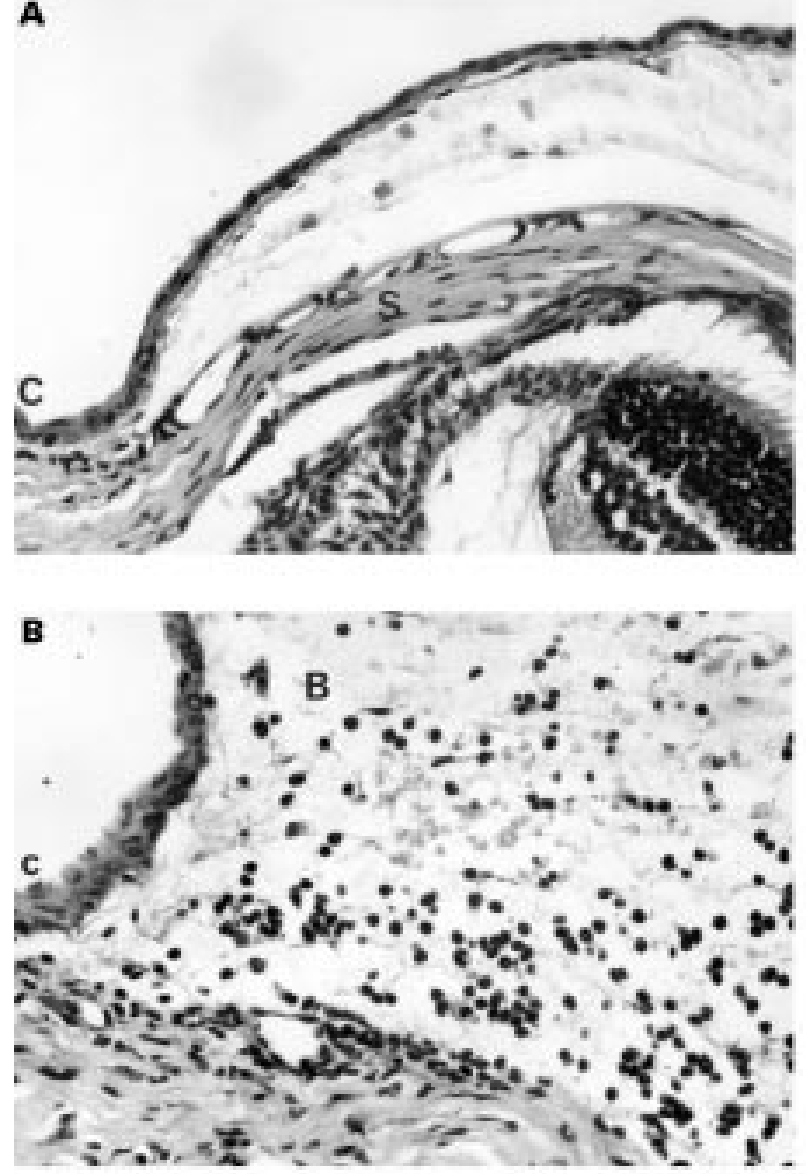

C

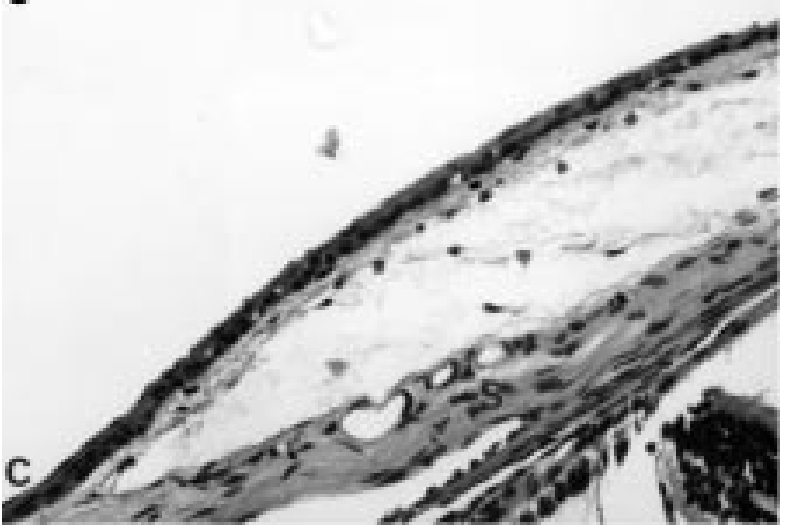

D

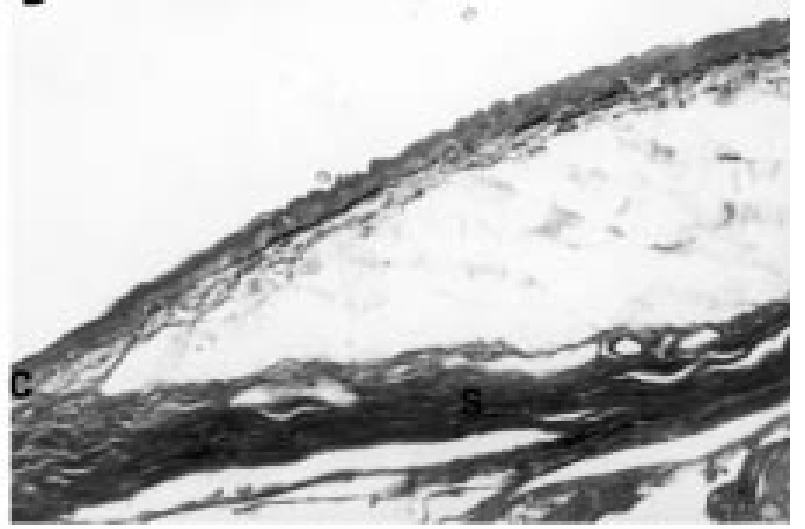

E

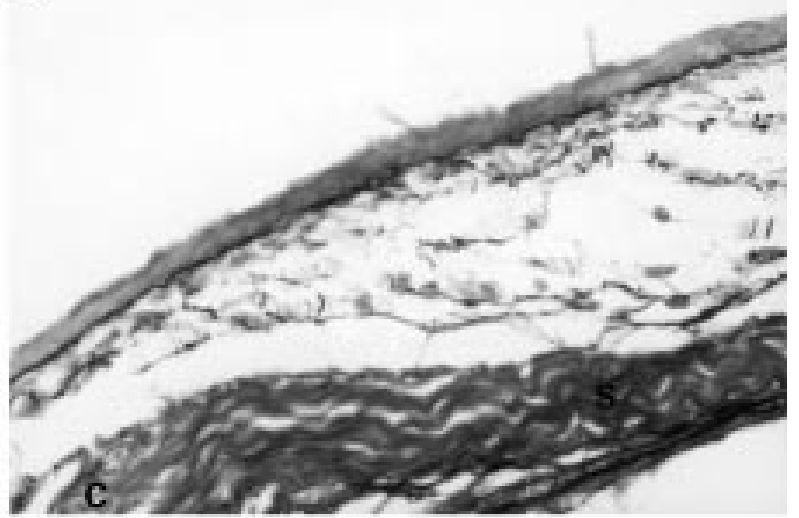

$\underline{\underline{\sigma}}$

○

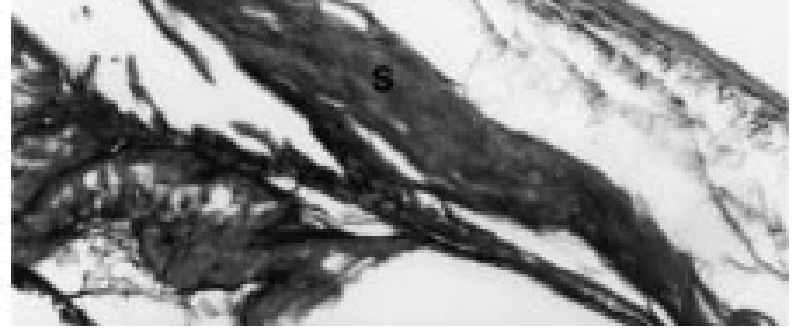

Figure 2 Histological evaluation of the cellular effects of the subconjunctival injection showed a typical wound healing response. Compared with the control eye (A), an initial influx of granulocytes (neutrophil polymorphonuclear leucocytes) was seen in association with considerable oedema of the subconjunctival tissue at day 1 in injected eyes (B). However, by day 14 (C) granulocytic and mononuclear activity had returned to control level. There was an increase in fibroblast number in the first several days after the injection, reaching a peak at day 3. Thereafter, fibroblast activity was found to decrease but did not ever return to the control eye activity level (haematoxylin and eosin stained $5 \mu m$ thick sections, $\times 80$ magnification. Cornea $=C$, $b l e b=B$, and sclera $=S$ ). Histological demonstration of extracellular matrix deposition in the subconjunctival bleb area using oxidation aldehyde-fuchsin stains. Early deposition of oxytalan and related fibres was demonstrated by the presence of dark, fibrillar fibres. Compared with control (D), increased deposition in injected eyes was seen at day 3 (E). An increased in amount and the degree of organisation of elastic related fibres was seen in injected eyes at day 14 compared with control $(F)$. (Aldehyde-fuchsin stained $5 \mu$ m thick sections, $\times 80$ magnification).

subconjunctival tissue with a peak in reaction at day 1 (Fig 2B), followed rapidly by a generalised inflammatory response. Figure $3 \mathrm{~A}$ illustrates the cellular profile within the subconjunctival bleb area, distinguishing between granulocytes, mononuclear cells, and fibroblasts. Granulocytic infiltration of the bleb area diminished and quickly returned to the control eye level by day
7. Mononuclear cells, identified morphologically as monocyte derived macrophages and lymphocytes, were initially present around blood vessels, and became the predominant cell type at day 2. Mononuclear cell activity decreased to the control eye level at day 14 .

The next wave of cellular activity seen was fibroblasts. There was an increase in total 

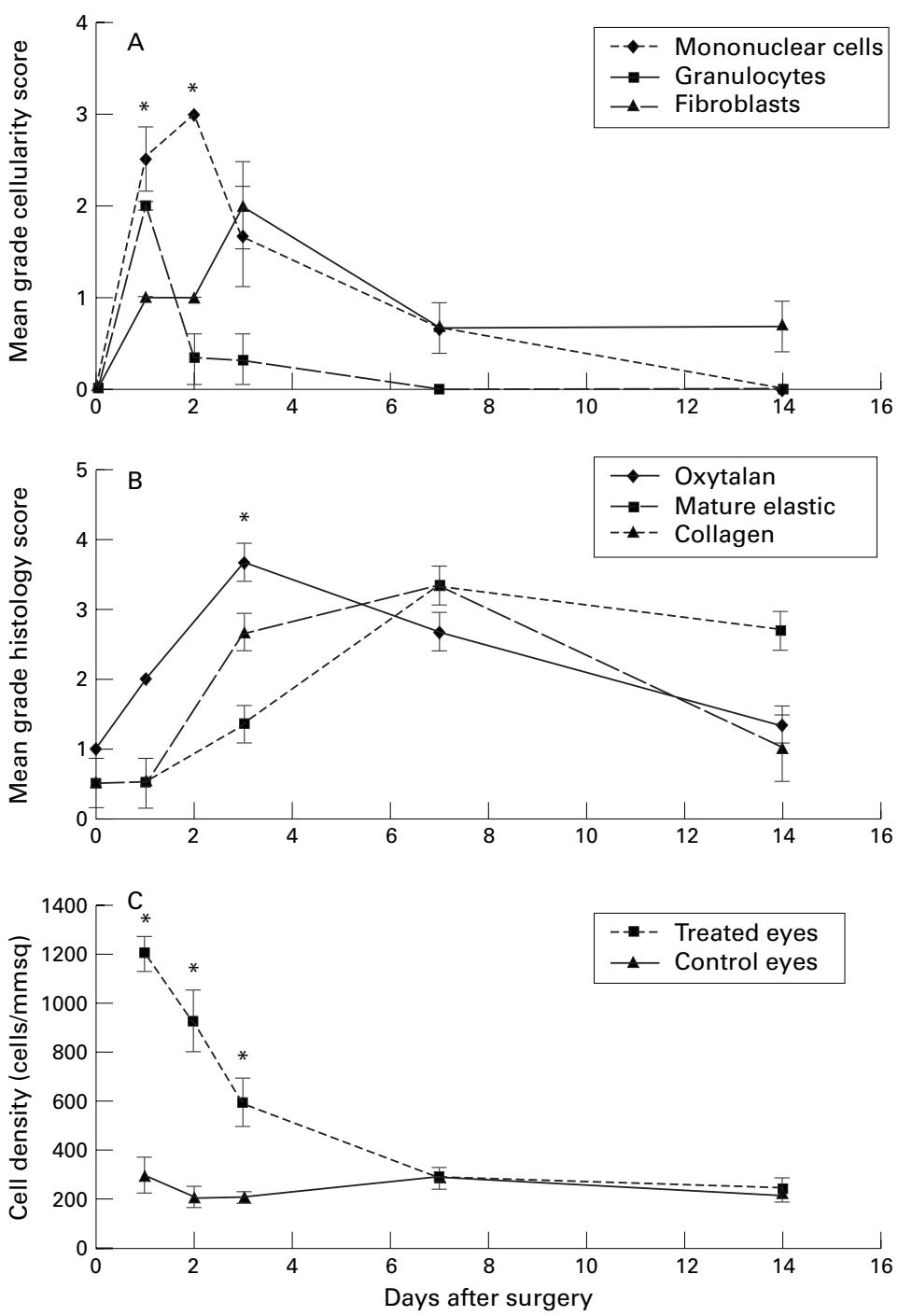

Figure 3 (A) Graph showing the cellular profile of mononuclear cells, granulocytes, and fibroblast activity in the bleb area over time, as graded by a masked observer (IAC). The consecutive peaks of these cell types demonstrate the classic wound healing response. (B) Graph showing the histochemical staining characteristics and the conjunctival scarring response. Newly laid extracellular matrix is demonstrated by the early deposition of oxytalan fibres, which later undergo maturation into elastic fibres. Collagen deposition occurs in association with wound remodelling, organisation, and contraction. (C) Graph showing the cellular density in injected eyes compared with their contralateral control eyes. $A$ fourfold increase in cellular density is seen at day 1. (All graphs show SEM error bars, with * indicating statistical significant differences between groups $p<0.05$.)

fibroblast number in the first several days, which reached a peak at day 3 (Fig 3A). Thereafter, fibroblast activity was found to decrease never returned to the control eye activity level. Statistical analysis using ANOVA showed significant differences between cell types (that is, mononuclear cells, granulocytes, and fibroblasts) on days 1 and $2, \mathrm{p}<0.05$ ).

Fibroblast activity was closely associated with the deposition of newly laid extracellular matrix, as evidenced by the presence of oxytalan fibres (dark fibrillar stain, Fig 2D-F). Maximal oxytalan demonstration was observed at day 3 (Fig 2D). This was closely linked to the deposition of newly laid collagen fibres as identified with the van Gieson stain (data not shown). Maturation of elastic fibres was first apparent at day 7 (Fig 3D). An increase in the number of mature elastic and collagen fibres was also present at this time, and there was evi- dence of continuing remodelling even at day 14 (Figs $2 \mathrm{~F}$ and 3B). Statistical analysis using ANOVA showed significant differences between the extracellular components on day 3 $(\mathrm{p}<0.05)$.

The histological study also included an evaluation of cellular density within the subconjunctival bleb area at the injection site. A fourfold increase in cellular density in the subconjunctival area in the eye that had been injected compared with the control eye was observed at day 1 (Fig 3C). After this initial peak, the cellularity decreased in an almost linear fashion to reach the density of the control eye by day 7 . Comparison between control and treated eyes revealed a statistically significant difference $(p<0.05)$ in total cellularity between the two groups.

\section{Discussion}

Our simple model of conjunctival scarring demonstrated a characteristic and reproducible healing response consisting of initial granulocyte invasion, followed by mononuclear cell infiltration and finally fibroblast involvement with deposition and remodelling of extracellular matrix.

The reaction produced by a simple, subconjunctival injection of PBS in the mouse eye is similar to that seen in wound healing as has been described elsewhere in the body. Most of the research that has been done in the field of wound repair and regeneration has focused on cutaneous wounds. The complex process of cutaneous wound repair has been simplified by dividing the sequence of events into three phases - the inflammatory, proliferative, and the remodelling phases. ${ }^{14}$ This division can be applied to wound healing throughout the body. ${ }^{15} 16$

The inflammatory phase is characterised by the influx of neutrophils and monocytes to the wound area. These white blood cells are induced by chemoattractants (for example, kallikrein, fibrin degradation products) which are released as products of the clotting and coagulation cascade. Neutrophil invasion occurs within hours of injury in skin wounds and is followed soon after by lymphocytes and macrophages. A similar pattern of inflammatory cell invasion was seen in our model and occurred in the first 2 days after surgery. The proliferative phase of wound healing in skin classically consists of re-epithelialisation, an increase in fibroblast activity and number, new vessel ingrowth, and the laying down of granulation tissue. In our model, all these processes were demonstrated except re-epithelialisation, as our model was subepithelial.

Fibroblasts play a central role in the proliferative and remodelling phases of wound healing. They mediate the processes of wound contraction and extracellular matrix deposition, degradation, and modification. Our conjunctival model showed increased fibroblast activity and extracellular matrix deposition between 3 and 7 days after subconjunctival injection. Thereafter, evidence of matrix remodelling and organisation of scar tissue was seen histologically up to 14 days after surgery. 
In skin, remodelling can continue for months and the amount of scar tissue formed has been shown to influenced by local environmental factors such as cytokines. For example, fetal wounds are believed to heal with minimal scarring because of the association of low levels of transforming growth factor $\beta 1$ (TGF- $\beta 1$ ). More recently, the effect of blocking TGF- $\beta 1$ and $\beta 2$ by the application of antibodies to adult cutaneous wounds demonstrated markedly reduced scarring. ${ }^{17} 18$

In animal models of filtration surgery, the conjunctival wound healing response has been found to be modified by the outflow of aqueous humour into the wound. The observation that the majority of filtration fistulas stay open has led to the hypothesis that aqueous humour may contain a substance that inhibits cell growth in vivo. ${ }^{19}$ Comparison between aqueous humour from cataract and glaucoma patients showed that aqueous from glaucoma patients was less inhibitory in its effect on in vitro conjunctival fibroblast cellular outgrowth..$^{20}$ Other studies have identified factors in aqueous humour, which may account for the differences seen in subconjunctival scarring following trabeculectomy. ${ }^{12} 2122$ Aqueous therefore plays an important role in the maintenance or failure of filtration blebs. However, it is still not fully understood how surgery affects aqueous constituents, and how the dynamic changes produced by aqueous flowing through the wound environment affects conjunctival scarring. By eliminating the involvement of aqueous in our model, we have characterised, in a longitudinal study, the conjunctival component of the scarring response.

Histological analysis of bleb failure in animal models of filtration surgery show that the time scale of the development of the conjunctival scarring response is similar to that seen in our mouse model. In the rat model, bleb failure was seen 7-19 days following filtration surgery. ${ }^{9}$ Failure in the rabbit, the most commonly used model in ophthalmic research, ${ }^{23}$ occurs around 14 days after surgery, ${ }^{25}$ as seen also in the owl monkey. ${ }^{35}$ The cellular profile in our model was also similar with respect to its consecutive peaks of neutrophils, monocytes, and fibroblast activation as described by previous authors. ${ }^{9} 26$

It is very interesting that a single subconjunctival injection of PBS in the mouse eye produces such a marked healing response. We know from similar experiments with other mouse strains that the inflammatory response following an intraocular injection is not strain specific. $^{27}$ The subconjunctival route is the commonest method of drug administration to the eye-for example, postoperative antibiotics and steroids. We used PBS because it is physiologically balanced and relatively innocuous. However, agents such as 5-FU and gentamicin, which are commonly given subconjunctivally, are known to have non-physiological $\mathrm{pHs}$ and cause epithelial toxicity. It is conceivable that the scarring response induced by these agents may be even more pronounced than that observed in our experiment. We have shown that even though the subconjunctival bleb dis- appears within 24 hours after the injection without any apparent macroscopic sequelae, as is similar to human eyes, there is still a wound healing reaction, albeit only at a histological level. In view of our findings there is a need for further work to address the important issue of subconjunctival drug delivery, especially because many ocular drugs are regularly given clinically via the subconjunctival route. Our study suggests that these drugs may be at risk of inducing a subconjunctival scarring response.

The rapid and aggressive healing response observed in the mouse makes it an ideal model in which to manipulate the wound healing process. The surgical procedure itself is easy and quick to perform, and unlike models of fistulising surgery, the effects of cytokines and chemical mediators within the aqueous are eliminated, and the reaction produced is localised purely to the subconjunctival level. In addition, because the mouse is so well characterised biologically, the ready availability of molecular reagents makes it particularly useful for further studies of growth factor protein and gene therapy and their potential role in wound healing manipulation.

In conclusion the response to subconjunctival injection in the mouse represents a simple, inexpensive and reliable model of conjunctival scarring in which it will be possible to study the effects of modulating agents such as antimetabolites, growth factors, and their antagonists.

This work was supported by Deutsche Forschungsgemeinschaft Re 1121/1-1 and Deutsche Retinitis Pigmentosa Vereinigung (MBR), the Wellcome Trust (MFC 048474), Moorfields Trustees, and the Medical Research Council (PTK)

1 Hitchings RA, Grierson I. Clinicopathological correlation in eyes with failed fistulizing surgery. Trans Ophthalmol Soc UK 1983;103:84-8.

2 Addicks EM, Quigley HA, Green WR, et al. Histologic characteristics of filtering blebs in glaucomatous eyes. Arch Ophthalmol 1983;101:795-8.

3 Desjardins DC, Parrish II RK, Folberg R, et al. Wound healing after filtrating surgery in owl monkeys. Arch Ophthalmol 1986;104:1835-9.

4 Khaw PT, Sherwood MB, MacKay SLD, et al. Five-minute treatment with fluorouracil, floxuridine, and mitomycin have long-term effects on human tenons capsule fibrohave long-term effects on human tenons
blasts. Arch Ophthalmol 1992;110:1150-4.

5 Gressel MG, Parrish RK, Folberg R. 5-Fluorouracil and glaucoma filtering surgery. Ophthalmology 1984;91:378-83.

6 Doyle JW, Sherwood MB, Khaw PT, et al. Intraoperative 5 -fluorouracil for filtration surgery in the rabbit. Invest Ophthalmol Vis Sci 1993;34:3313-19.

7 Bergstrom TJ, Wilkinson WS, Skuta GL, et al. The effects of subconjunctival mitomycin-C on glaucoma filtration surgery in rabbits. Arch Ophthalmol 1991;109:1725-30.

8 Pfeiffer RL, Lipper S, Merrit JC, et al. Myofibroblasts in healing of filtering wounds in rabbit, dog, and cat. Glaucoma 1981;3:277-80.

9 Sheridan CM, Unger WG, Ayliffe W, et al. Macrophages during fibrosis following scleral fistulising surgery in a rat model. Curr Eye Res 1996;15:559-68.

10 Miller MH, Grierson I, Unger WI, et al. Wound healing in an animal model of glaucoma fistulating surgery in the rabbit. Ophthalmic Surg 1989;20:350-7.

11 Miller AD. Retrovirus packaging cells. Hum Gene Ther 1990;1:5-14.

12 Joseph JP, Grierson I, Hitchings RA. Chemotactic activity of aqueous humor. A cause of failure of trabeculectomies? Arch Ophthalmol 1989;107:69-74.

13 Alexander RA, Hiscott P, McGalliard J, et al. Oxytalan fibres in proliferative vitreoretinopathy. Ger f Ophthalmol 1992;1: 382-7.

14 Kirsner RS, Eaglstein WH. The wound healing process. Wound Healing 1993;11:629-40.

15 Khaw PT, Occleston NL, Schulz GS, et al. Activation and suppression of fibroblast activity. Eye 1994;8:188-95.

16 Clark RAF, Henson MP. The molecular and cellular biology of wound repair. New York: Plenum Press, 1988.

17 Shah M, Foremann DM, Ferguson MWJ. Neutralising antibody to TGF-beta 1,2 reduces cutaneous scarring in adult rodents. F Cell Sci 1994;107:1137-57. 
18 Shah M, Foremann DM, Ferguson MWJ. Neutralisation of TGF-beta 1 and TGF-beta 2 or exogenous addition of TGF-beta 3 to cutaneous rat wounds reduces scarring. $f$ Cell Sci 1995;108:985-1002.

19 Snell AC. Wound healing of the iris. Am f Ophthalmol 1956; 41:499-505.

20 Herschler J, Claflin AJ, Fiorentino G. The effect of aqueous humor in the growth of subconjunctival fibroblasts in tissue culture and its implication for glaucoma surgery. Am $\mathcal{F}$ Ophthalmol 1980;89:245-9.

21 Mehra KS, Dube B, Dube RK. Fibrinolytic activity in blood and aqueous humour in glaucoma. Ind f Ophthalmol 1983; 31:827-9.

22 Radius RL, Herschler J, Claflin A, et al. Aqueous humor changes after experimental filtering surgery. Am f Ophthalmol 1980;89:250-4.
23 Lindsey JR, Fox RR. Models in ophthalmology and vision research. In: Pfeifer RL, Pohm-Thorsen L, Corcoran K, eds. The biology of the labor
Academic Press 1994:424-6.

24 Prince JH.The rabbit in eye research. Springfield, IL: Charles C Thomas, 1964.

25 Miller MH, Joseph NH, Ennis KW, et al. An animal model of filtration surgery. Trans Ophthalmol Soc UK 1985;104: 893-7.

26 Costa VP, Speath GL, Eiferman RA, et al. Wound healing modulation in glaucoma filtration surgery. Ophthalmic Surg 1993;24:152-70.

27 Reichel MB, Ali RR, Baker D, et al. Co-injection of adenovirus expressing CTLA4-Ig prolongs adenovirallymediated lacZ reporter gene expression in the mouse retina. Invest Ophthalmol Vis Sci 1997;38(Suppl): S259. 